\title{
Development of a Finger Extension Assistance Splint for a Hemiplegic Upper Limb After Stroke - A Case Report
}

\author{
Ryo Sakai ${ }^{1}$, Makoto Haraguchi ${ }^{2}$, Masayuki Kanamori ${ }^{3}$, \\ Katsunori Yamada ${ }^{3}$, Keiji Ishida ${ }^{1}$, Yasutaka Kobayashi ${ }^{1}$ \\ ${ }^{1}$ Department of Rehabilitation, Faculty of Health Science, Fukui Health Science University, Japan \\ ${ }^{2}$ Department of mechanical engineering, Faculty of Engineering, Osaka Institute of Technology, Japan \\ ${ }^{3}$ Department of Rehabilitation Occupational Therapy, Fukui General Hospital, Japan
}

\begin{abstract}
Background: Paralysis of the upper limb after stroke affects daily life. Our objective was to examine the clinical effects of our newly developed finger extension splint, which was made by specially processing a shape memory alloy, in a chronic stroke patient.

Design: Comparison of the clinical effects of the splint use pre- and post-treatment.

Patient: A 70-year-old man who developed right thalamus hemorrhage 15 years previously presented with right hemiplegia.

Intervention: Training was performed 30 minutes/day for 10 weekdays for the fingers on the paralyzed side. Taskoriented training, such as block stacking, was performed.

Results: Upper limb function evaluation results (pre vs post-treatment): grip strength, $9.3 \mathrm{vs} 12.1 \mathrm{~kg}$; upper-extremity subtest of the Fugl-Meyer Assessment of Motor Function, 42 vs 54 points; Wolf Motor Function Test (functional ability scale), 47 vs 50 points; time, 389.2 vs 308.2 seconds, and the Box and Block Test: 11 vs 15 and 8 vs 15 with and without the splint, respectively.

Conclusion: When training was conducted using this splint, improvement of upper limb function was observed.
\end{abstract}

Keywords: stroke, arm function, splint

(Asian J Occup Ther 16: 83-86, 2020)

\section{Introduction}

Stroke causes motor paralysis in the unilateral upper and lower limbs due to ischemic or hemorrhagic lesions in the brain. Increased muscle tension (spasticity) is difficult to treat and greatly affects activities of daily living (ADL).

Orthopedic therapy is used to apply continuous tension to muscles and joints, mainly to prevent the progression and deformation of joint contractures associated with spasticity. Although previous studies report

Received: 27 August 2019, Accepted: 16 September 2020

Corresponding to: Ryo Sakai, Department of Rehabilitation, Faculty of Health Science, Fukui Health Science University, 55-13-1 Egami, Fukui City, Fukui 910-3190, Japan

e-mail: r-sakai@fukui-hsu.ac.jp

C2020 Japanese Association of Occupational Therapists that static splints may to be sufficiently effective in preventing joint contractures due to spasticity $[1,2,3]$, attenuation of the effects of spasticity was reported with the use of dynamic splints, such as dorsal support functional braces [4], dynamic hand splints [5], and spider splints [6].

The degree of motor paralysis and spasticity varies between individuals, and it is important to use a splint that is appropriate for the patient's condition [7]. Previous studies have shown that each joint needs to be properly tensioned, but most dynamic splints pull the entire finger, including the interphalangeal (IP) and metacarpophalangeal (MP) joints. Thus, proper tension is not applied to each joint. We treated patients by prescribing that they wear spider splints, but we found that, in some patients, the MP joints were excessively extended due to the effect of the balance of muscle tension in the internal and external muscles. Therefore, as the first 

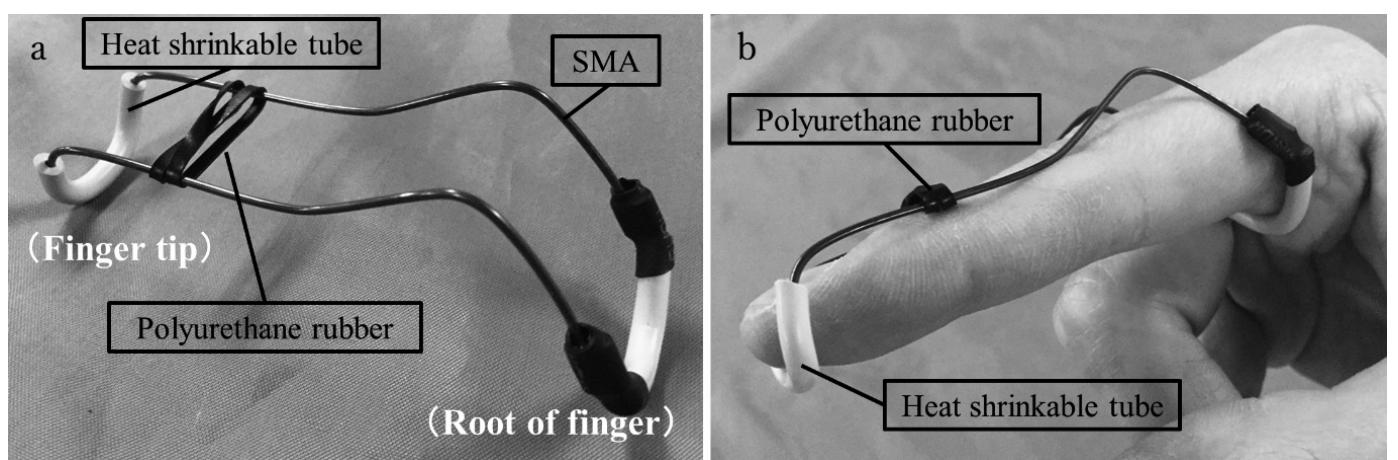

Fig. 1. Structure of the finger extension assistance splint. (a) Details of the structure of the finger extension splint. (b) Splint installation. $\mathrm{SMA}=$ Shape memory alloy.
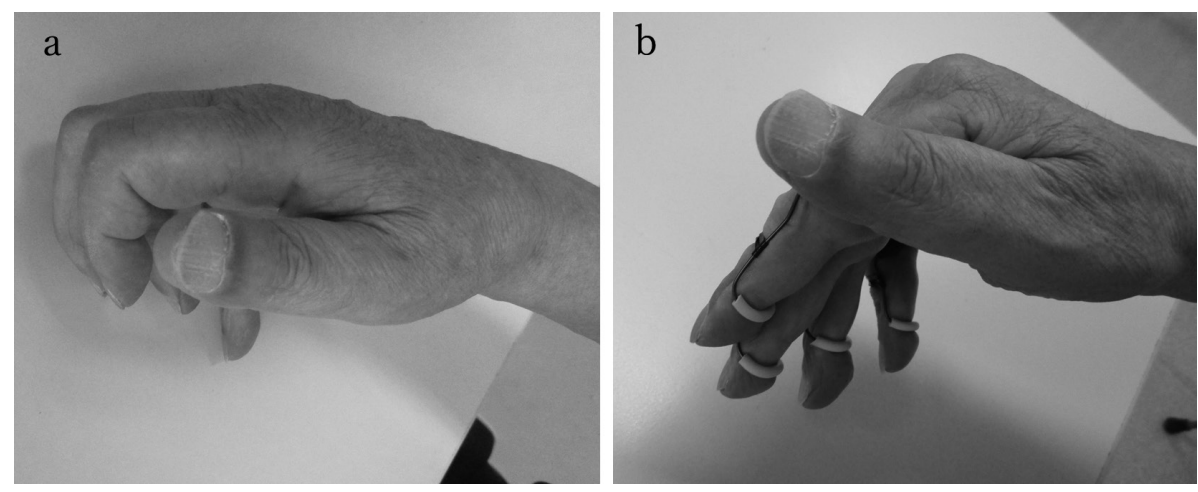

Fig. 2. Comparison of finger extension movement with and without the splint. State of finger extension without the splint (a) and with the splint (b).

step, we have developed a splint that responds to the degree of paralysis and spasticity in individual patients and provides tension to the IP joint independently. In this study, we used finger extension assistance splints for a chronic stroke patient and report clinical effects based on a comparative pre- and post-treatment study.

\section{Methods}

\subsection{Structure of orthosis and method of fabrication}

We developed a new splint that can support finger extension in hemiplegic patients with spasticity (Fig. 1). This device uses a shape memory alloy (SMA) wire with superelastic properties. When the finger is flexed, the SMA with a bent beam shape is deformed, and an assist force in the extension direction is generated in the distal IP and proximal IP joints. To reduce pain in the contact area, a heat-shrinkable tube with high cushioning properties is placed at the tip and base of the finger. The material at the base of the finger is a steel wire, which can handle various finger thicknesses. The length of the splint can be altered to fit the individual's fingers. Because the degree of spasticity varies between individ- uals, the assist power of this device can be adjusted by changing the diameter of the SMA.

\subsection{Participant}

A 70-year-old man, who developed right thalamus hemorrhage 15 years earlier, presented with right hemiplegia. Range of motion was reduced in his upper right hand and fingers due to spasticity and muscle shortening, although he could perform ADL independently.

\subsection{Interventions and outcomes}

The patient trained for 10 days of 2 weeks, 30 minutes/day. Task-oriented training was performed according to previous research $[8,9]$, with the finger extension splint attached to II-V fingers (Fig. 2). The patient performed a task of moving beanbags and wooden blocks as task-oriented training. The difficulty level of the task was gradually increased according to the success rate of the task, while providing feedback regarding the task execution time to the patient. Evaluation of upper limb function was performed pre- and post-intervention and included grip strength, upper-extremity subtest of the Fugl-Meyer Assessment of Motor Function (FMA-UE), 


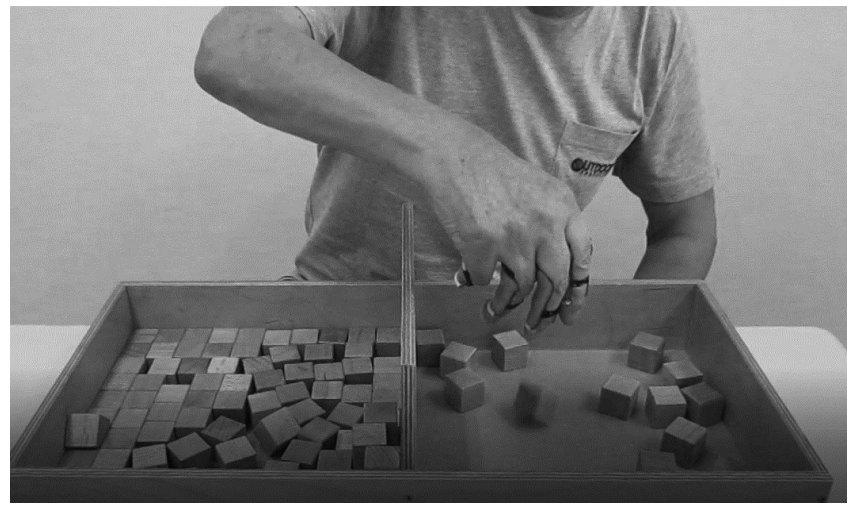

Fig. 3. The patient is wearing the splint and is able to move the blocks (Box and Block Test).

Wolf Motor Function Test (WMFT), Functional Ability Scale (FAS)/Time, and the Box and Block Test (BBT). BBT measurements were obtained twice, with and without the splint (Fig. 3). Evaluation of spasticity was with the Modified Ashworth Scale-elbow, finger (MAS-elbow, finger).

This research was conducted with the approval of the Nittazuka Medical Welfare Center Ethics Review Committee (ethics examination number: Shinrin 2871). We explained the study to the subject in writing and verbally, and obtained consent. The authors declare no conflict of interest.

\section{Results}

Upper limb function evaluation results, pre- vs posttreatment, were: grip strength, 9.3 vs $12.1 \mathrm{~kg}$; FMAUE, 42 vs 54 points; WMFT FAS, 47 vs 50 points; time, 389.2 vs 308.2 seconds; Motor Activity Log-Amount of Use (MAL-AOU), 0.33 vs 0.41 points; Motor Activity Log-Quality of Movement (MAL-QOM), 0.30 vs 0.34 points and BBT, 11 vs 15 (when wearing a splint) and 8 vs 15 (when not wearing a splint) (Table 1). There was no change in MAS.

\section{Discussion}

In this case, although flexion of the fingers was possible, voluntary extension movement was weak, and it was difficult to release an item once grasped. When training was conducted using this splint, improvement of upper limb function was observed. Before and after treatment, the amount of improvement for the patient was as follows: grip strength was $2.8 \mathrm{~kg}$; the FMA-UE and WMFT-FAS were 12 and 3 points, respectively; the WMFT-time was 81 seconds, the MAL-AOU and MAL-
Table 1 Upper limb function evaluation results before and after training with the splint

\begin{tabular}{lccc}
\hline \multicolumn{1}{c}{ Outcome } & Pre-treatment & Post-treatment & $\begin{array}{c}\text { Change } \\
\text { value }\end{array}$ \\
\hline Grip (kg) & 9.3 & 12.1 & $\mathbf{2 . 8}$ \\
FMA-UE & 42 & 54 & $\mathbf{1 2}$ \\
MAS-elbow & 1 & 1 & 0 \\
MAS-finger & 3 & 3 & 0 \\
WMFT-FAS & 47 & 50 & $\mathbf{3}$ \\
WMFT-times (second) & 389.2 & 308.2 & $\mathbf{8 1}$ \\
MAL-AOU & 0.33 & 0.41 & $\mathbf{0 . 0 8}$ \\
MAL-QOM & 0.30 & 0.34 & $\mathbf{0 . 0 4}$ \\
BBT: without splint & 8 & 15 & $\mathbf{7}$ \\
BBT: with splint & 11 & 15 & $\mathbf{4}$ \\
\hline
\end{tabular}

FMA-UE = Upper-extremity subtest of the Fugl-Meyer Assessment of Motor Function; MAS = Modified Ashworth Scale; WMFT = Wolf Motor Function Test; FAS = Functional Ability Scale; MAL = Motor Activity Log; AOU = Amount of Use; QOM = Quality of Movement; $\mathrm{BBT}=$ Box and Block Test

Outcomes with improvements post-treatment are indicated in bold.

QOM were 0.08 and 0.04, respectively; and the BBT was 7. In comparison, previous studies have reported a grip strength, according to the Minimum Clinically Important Difference (MCID), of $2.9 \mathrm{~kg}$, an FMA-UE of 4.25-7.25, 1 point for WMFT-FAS, a WMFT-time of 19 seconds, 0.5 points for MAL-AOU and QOM, and a BBT of $5.5[10,11,12,13]$. Thus, although the grip strength and the frequency of paralyzed hand use in daily life did not exceed the MCID, the changes in motor function evaluations such as FMA-UE, WMFT and BBT did exceed the MCID, which was clinically sufficient. When this splint is used in conjunction with task-oriented training, bending and stretching exercises of the fingers are repeated, and it is considered that motor learning effects are produced $[5,9,14,15]$. These studies suggest that hand splints can be used for motor learning training and improving neural plasticity, such as motor cortex priming and reorganization in the brain. In addition, by performing task-oriented training under the above-mentioned environment, we believe that the treatment effect will not only extend to finger movements but also to the entire upper limb. The treatment effect in this case was not limited to the motor learning effect of finger extension; notably, improvements in grip strength and object handling ability were observed.

Dynamic splints increase muscle tissue stability, limit unnecessary movements that impair hand function, supplement weak muscles, and maintain the natural anatomical alignment of the hand [16]. Unlike the conventional splint, our splint allows fine adjustment of tension and size for each finger, provides tension parallel to the extension direction of the finger, and provides appropriate assistance along the normal structure of the 
finger. In addition, our splint is smaller and lighter, and has the advantage of being less noticeable in everyday life situations. We believe that the development of splints with a structure like ours that follows the anatomy and anatomical functions of the finger is necessary.

\section{Study limitations and future research}

This study includes one case. We recommend further large-scale studies to investigate the state of muscle tone more objectively and quantitatively using physiological tests, such as evoked electromyography. In addition to this splint, we are developing a splint that provides separate tension to the MP joint. In the future, we will study the structure and methodology that provide proper tension to the distal interphalangeal and proximal interphalangeal joints, respectively.

\section{Acknowledgments}

We thank the students at Fukui Institute of Technology who helped develop this splint, and the staff at the rehabilitation section of Fukui General Hospital.

\section{References}

[1] Blackmore AM, Garbellini SA, Buttigieg P, Wells J. A systematic review of the effects of soft splinting on upper limb function in people with cerebral palsy. An AACPDM Evidence Report. Initial publication in AACPDM Org. October 2006.

[2] Choi JB, Ma SR, Song BK. The effect of resting hand splint on hand pain and edema among patients with stroke. J Ecophysiol Occup Hlth. 2016; 16(1\&2): 37-41.

[3] Lannin NA, Cusick A, McCluskey A, Herbert RD. Effects of splinting on wrist contracture after stroke: a randomized controlled trial. Stroke. 2007; 38(1): 111-6.

[4] McPherson JJ, Becker AH, Franszczak N. Dynamic splint to reduce the passive component of hypertonicity. Arch Phys Med Rehabil. 1985; 66(4): 249-52.

[5] Chang W-D, Lai P-T. New design of home-based dynamic hand splint for hemiplegic hands: a preliminary study.
J Phys Ther Sci. 2015; 27(3): 829-31.

[6] Tanabe H, Nagao T, Tanemura R. Application of constraint-induced movement therapy for people with severe chronic plegic Hand. Asian J Occup Ther. 2011; 9(1): 7-14.

[7] Hoffman HB, Blakey GL. New design of dynamic orthoses for neurological conditions. NeuroRehabilitation. 2011; 28(1): 55-61.

[8] Nelles G, Jentzen W, Jueptner M, Müller S, Diener HC. Arm training induced brain plasticity in stroke studied with serial positron emission tomography. Neuroimage. 2001; 13(6): 1146-54.

[9] Taub E, Uswatte G, Bowman MH, Mark VW, Delgado A, Bryson C, et al. Constraint-induced movement therapy combined with conventional neurorehabilitation techniques in chronic stroke patients with plegic hands: a case series. Arch Phys Med Rehabil. 2013; 94(1): 86-94.

[10] Lang CE, Edwards DF, Birkenmeier RL, Dromerick AW. Estimating minimal clinically important differences of upper-extremity measures early after stroke. Arch Phys Med Rehabil. 2008; 89(9): 1693-700.

[11] Page SJ, Fulk GD, Boyne P. Clinically important differences for the upper-extremity Fugl-Meyer scale in people with minimal to moderate impairment due to chronic stroke. Phys Ther. 2012; 92(6): 791-8.

[12] van der Lee JH, Wagenaar RC, Lankhorst GJ, Vogelaar TW, Devillé WL, Bouter LM. Forced use of the upper extremity in chronic stroke patients: results from a singleblind randomized clinical trial. Stroke. 1999; 30(11): 2369-75.

[13] Chen H-M, Chen CC, Hsueh I-P, Huang S-L, Hsieh C-L. Test-retest reproducibility and smallest real difference of 5 hand function tests in patients with stroke. Neurorehabil Neural Repair. 2009; 23: 435-40.

[14] Prakash J, Mondam S, Srinivasan M, Abbina AK. Effectiveness of dynamic wrist splint on deltoid muscle activity using functional task practice in post stroke patients. Int J Physiother Res. 2013; 2(2): 30-5.

[15] Pitts DG, O'Brien SP. Splinting the hand to enhance motor control and brain plasticity. Top Stroke Rehabil. 2008; 15(5): 456-67.

[16] Radomski LCM. Occupational therapy for physical dysfunction. 6th ed. Baltimore, MD: Wolters Kluwer/ Lippincott Williams \& Wilkins; 2007. 